\title{
Wavelength scaling of high-harmonic generation efficiency close to the multiphoton ionization regime
}

\author{
Chien-Jen Lai, ${ }^{1}$ Giovanni Cirmi, ${ }^{1,2}$ Shu-Wei Huang, ${ }^{1}$ Eduardo Granados, ${ }^{1}$ Kyung-Han Hong, ${ }^{1}$ Jeffrey Moses, ${ }^{1}$ \\ Phillip Keathley, ${ }^{1}$ Siddharth Bhardwaj, ${ }^{1}$ and Franz X. Kärtner ${ }^{1,2}$ \\ ${ }^{I}$ Department of Electrical Engineering and Computer Science and Research Laboratory of Electronics, \\ Massachusetts Institute of Technology (MIT), Cambridge, Massachusetts 02139, USA, \\ ${ }^{2}$ Center for Free-Electron Laser Science, DESY and Department of Physics, Hamburg University, Hamburg, Germany \\ cjlai@mit.edu
}

\begin{abstract}
Our experiment shows a less dramatic wavelength scaling of high harmonic generation efficiency between the tunneling and the multiphoton ionization regimes, which can be explained by a modified three-step model with complex ionization time.

OCIS codes: (190.2620) Harmonic generation and mixing; (270.6620) Strong-field processes
\end{abstract}

\section{Introduction}

The wavelength scaling of HHG efficiency has been studied in many theoretical works [1] and experiments [2] using infrared (IR) driver wavelengths. For driver wavelengths shorter than $800 \mathrm{~nm}$, a systematic experiment employing a dense set of wavelengths is still necessary to experimentally map out the detailed wavelength scaling relation of the single-atom efficiency (SE). For short-wavelength drivers, the three-step model (TSM) needs to be carefully applied because the semi-classical picture in the conventional TSM assumes the Keldysh parameter $\gamma$ is much less than one and neglects its influence. Therefore, we study the wavelength scaling of the SE driven by visible wavelengths between 500 and $800 \mathrm{~nm}$ in the transition between the tunneling and the multiphoton ionization with $\gamma \approx 1$. Our experiment shows a less dramatic wavelength scaling than the conventional IR-driven HHG, and this discrepancy can be explained by our modified TSM that employs a consistent approach including both the complex ionization time [3] and the non-adiabatic (NA) ionization [4]

\section{Experiment Result}

In the experiment, the driver sources were a Ti:sapphire amplifier and our OPA system that generated a signal continuously tunable between $470 \mathrm{~nm}$ and $650 \mathrm{~nm}$ with 34 39 fs pulse duration [5]. Since we were interested in the scaling of the SE, the temporal and spatial conditions of these driver pulses were carefully controlled to minimize the interference from macroscopic factors. The peak intensities of the tested driver wavelengths were fixed at $(2.7 \pm 0.2) \times 10^{14} \mathrm{~W} / \mathrm{cm}^{2}$, corresponding to Keldysh parameters between 0.7 and 1.1. The HHG medium was an Ar gas jet whose position relative to the driver pulse focus was optimized for phase-matching of the high harmonic $(\mathrm{HH})$ signal. We optimized and compared the harmonic peaks around $32 \mathrm{eV}$ from the 524, 589, 633, and $800 \mathrm{~nm}$ driver wavelengths. The HHG spectra are shown in Fig. 1(a), and the optimized harmonics that we compared in our study are indicated by arrows. We compare the total high harmonic yield within each specific harmonic peak as plotted in Fig. 1(b). The linear fitting on the log-log scale shows a wavelength scaling of $\lambda^{-(4.7 \pm 1.0)}$.
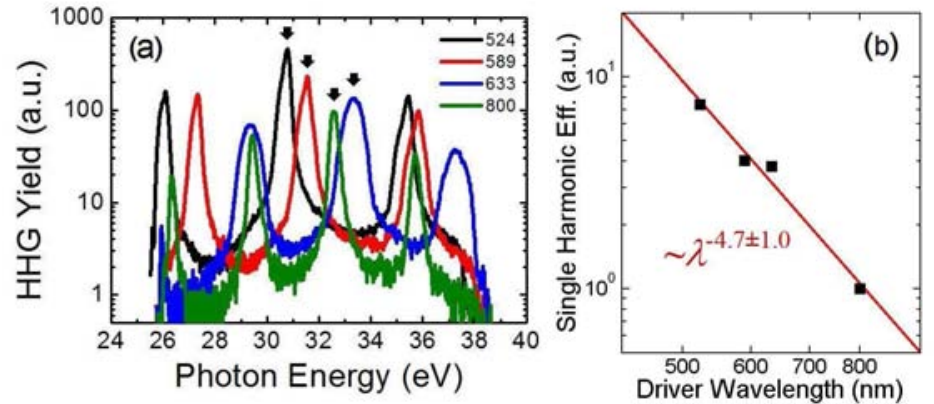

Fig. 1 (a) The HHG experiment spectra, where the legend shows the driver wavelengths in nm, and the arrows indicate the optimized phase-matched harmonics selected for our comparison. (b) The integrated signal of the individual harmonics indicated by the arrows in (a) on log-log scale. The fitting shows a $\lambda^{-4.7 \pm 1.0}$ wavelength scaling of HHG single harmonic efficiency.

For IR driver wavelengths, a wavelength scaling relation of $\lambda^{-(5 \sim 6)}$ has been reported by comparing the total HHG yield within a certain range of photon energy from driver pulses with the same number of electric field cycles [1]. 
However, this way of comparison is not suitable for HHG experiments driven by short wavelengths because the HHG spectra have narrower bandwidths and sparser harmonics. It is more appropriate to compare the single harmonic yield near some fixed photon energy with the same driver pulse energy. Conformed to the latter definition, the scaling relation should be $\lambda^{-(7 \sim 8)}$. With the same definition of HHG efficiency, our $\lambda^{-4.7 \pm 1.0}$ experiment result is less dramatic, and we attribute this difference to the larger Keldysh parameter and the deviation from a pure tunneling process, as supported by the calculation with our modified TSM below.

\section{Numerical Comparison}

Assuming a negligible $\gamma$, the conventional TSM neglects the ionization potential in the calculation of the saddle point of the ionization time and uses the quasi-static ADK formula to calculate the ionization rate [6]. It has been shown that the ADK formula can be replaced by the NA ionization for more accurate calculation of the ionization rate [7]. To further improve the TSM, we include the ionization potential and calculate the exact saddle point of the ionization time, which is generally a complex number. Fig. 2 compares the experimental data with the conventional TSM, the modified TSM, and the time-dependent Schrödinger equation (TDSE) results. While the conventional TSM shows a $\lambda^{-7.1}$ scaling (Fig. 2(b)), the modified TSM shows a scaling relation of $\lambda^{-4.8}$, close to the experiment result $\lambda^{-4.7}$. The numerical result from the TDSE calculation (Fig. 2(d)) is also in good agreement with our modified TSM and experiment. The difference of the scaling relation between the two TSM comes from the wavelength scaling of the traveling time of the ionized electron, which affects, among other things, the degree of quantum diffusion. In the conventional TSM, the traveling time between the ionization and the recombination is real and linearly proportional to the driver wavelength, while in the modified TSM, the traveling time depends on the driver wavelength and the Keldysh parameter $\gamma$, and scales with the driver wavelength in a different way.
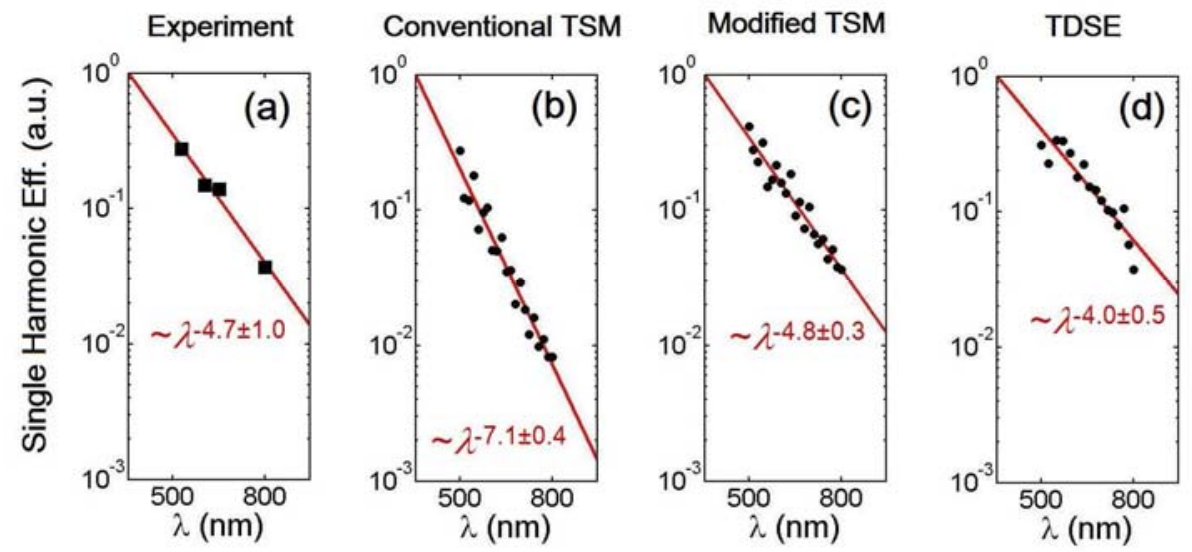

Fig. 2 Wavelength scaling of single harmonic efficiency (a) measured by the experiment, and calculated by (b) the conventional TSM, (c) the modified TSM, and (d) the TDSE. The horizontal axes show the driver wavelength $\lambda$ (on a log scale), and the vertical axes show the efficiency of the harmonic near $32 \mathrm{eV}$.

\section{Conclusion}

We study the wavelength scaling of high order harmonic generation efficiency with visible driver wavelengths in the transition between the tunneling and the multiphoton ionization regimes where the Keldysh parameter is around unity. Our experiment shows a less dramatic wavelength scaling of efficiency than the conventional case for IR driver wavelengths, and it is well explained by a modified TSM for an increased Keldysh parameter that employs the NA ionization rate and a complex ionization time.

\section{References}

[1] J. Tate et al, "Scaling of Wave-Packet Dynamics in an Intense Midinfrared Field," Phys. Rev. Lett. 98, 013901 (2007).

[2] A. D. Shiner et al, "Wavelength Scaling of High Harmonic Generation," Phys. Rev. Lett. 103, 073902 (2009).

[3] L. Torlina and O. Smirnova, "Time-dependent analytical R-matrix approach for strong-field dynamics. I. One-electron systems," Phys. Rev. A 86, 043408 (2012).

[4] G. L. Yudin and M. Yu. Ivanov, "Nonadiabatic tunnel ionization: Looking inside a laser cycle,” Phys. Rev. A 64, 013409 (2001).

[5] G. Cirmi et al, "Cut-off scaling of high-harmonic generation driven by a femtosecond visible optical parametric amplifier," J. Phys. B 45, 205601 (2012).

[6] E. L. Falcão-Filho et al, “Analytic scaling analysis of high harmonic generation conversion efficiency,” Opt. Express 17, 11217 (2009).

[7] V.-M. Gkortsas et al, "Interplay of multiphoton and tunneling ionization in short-wavelength-driven high-order harmonic generation," Phys. Rev. A 84, 013427 (2011). 